Cita: Pulido, J. J.; Merino, A.; Sánchez-Oliva, D.; Tapia, M. A.; y García-Calvo, T. (2019).

Efectos de un programa de intervención sobre el estilo interpersonal de entrenadores de fútbol base. Cuadernos de Psicología del Deporte, Vol 19(1), 32-49

\title{
Efectos de un programa de intervención sobre el estilo interpersonal de entrenadores de fútbol base
}

\section{Effects of an intervention program on the interpersonal style of football coaches}

\section{Efeitos de um programa de intervenção sobre o estilo interpessoal dos treinadores de futebol}

\author{
Pulido, J. J. ${ }^{1}$, Merino, A. ${ }^{1}$, Sánchez-Oliva, D. ${ }^{1,2}$, Tapia, M. A. ${ }^{1}$, y García-Calvo, T. ${ }^{1}$ \\ ${ }^{1}$ Facultad de Ciencias del Deporte. Universidad de Extremadura; ${ }^{2}$ Facultad de Ciencias de la \\ Educación. Universidad de Cádiz.
}

\section{RESUMEN}

El objetivo de este estudio era analizar los efectos de un programa de formación sobre la conducta verbal de entrenadores de fútbol base sin formación federativa y académica relacionada con la actividad física y el deporte. El programa estaba basado en el fomento de estrategias motivacionales y metodológicas para optimizar las necesidades psicológicas básicas de autonomía, competencia y relaciones sociales de los deportistas, aumentando las conductas relacionadas a un estilo interpersonal de apoyo y, reduciendo las conductas asociadas a un estilo interpersonal controlador. Participaron ocho entrenadores distribuidos intencionadamente en dos grupos: grupo experimental $(n=4)$ y grupo control $(n=4)$. Tras la observación del estilo interpersonal de los entrenadores (pre y post) mediante un instrumento creado a propósito para el estudio, no se obtuvieron diferencias significativas entre los dos grupos, aunque los resultados del grupo experimental demostraron una tendencia positiva entre la medida pre-intervención y la post-intervención. Por tanto, el programa resulta eficaz pero no en la medida que se esperaba, por lo que es preciso seguir investigando en esta línea de trabajo.

Palabras clave: conducta verbal, estilo interpersonal, intervención, necesidades psicológicas básicas, fútbol.

\section{ABSTRACT (150 words) (same order that title)}

The main objective of this study was to analyze the effects of a training program on the verbal behavior of unskilled youth football coaches without training and academic qualification (related with physical and sport training). The program was based on the promotion of motivational and methodological strategies with the purpose of optimizing the athletes' basic psychological needs of autonomy, competence and relatedness, through an interpersonal style of support and, in turn, reducing the controlling behaviors. Participated eight coaches intentionally distributed in two groups: experimental group $(n=4)$ and control group $(\mathrm{n}=4)$. After observing of the interpersonal style of the coaches (pre and post) by an instrument created on purpose for the study, there was not significant differences in the extent post-intervention 
between groups, regarding the repeated measures analysis of the experimental group there are improvements between pre-intervention and post-intervention measure. Therefore, the program is effective but not to the extent expected, so it is necessary to continue research in this line of work.

Keywords: verbal behavior, intervention program, basic psychological needs, football training.

\section{RESUMO}

O objetivo deste estudo foi analisar os efeitos de uma intervenção no comportamento verbal de treinadores sem formação federativa e acadêmica relacionada à atividade física e desporto. O programa foi baseado na promoção de estratégias motivacionais e metodológicas para otimizar as necessidades psicológicas básicas dos atletas de autonomia, competência e relações sociais, através de um estilo de apoio interpessoal e reduzindo os comportamentos controladores. Oito treinadores participaram intencionalmente e foram distribuídos em dois grupos: grupo experimental $(n=4)$ e grupo de controlo $(n=4)$. Depois de observar o estilo interpessoal dos treinadores (pré e pós) através de um instrumento criado para o propósito do estudo, não foram encontradas diferenças significativas entre os dois grupos, embora os resultados do grupo experimental tenham mostrado uma tendência positiva entre a medida pré-intervenção e a pós-intervenção. Portanto, o programa é eficaz, mas não na medida esperada, sendo por isso necessária mais investigação neste âmbito.

Palavras chave: comportamento verbal, programa formativo, necessidades psicológicas básicas, futebol.

\section{INTRODUCCIÓN}

En el deporte en etapas de iniciación existen diferentes agentes socializadores que van a influir en el desarrollo deportivo y personal de los jóvenes que lo practican: padres, entrenadores, compañeros, árbitros... (Ramis, Torregrosa, Viladrich, y Cruz, 2013). Centrándonos en la figura del entrenador, se estima que, durante las etapas deportivas iniciales, un joven de entre $9 \mathrm{y}$ 16 años comparte más de 2.500 horas con su entrenador (Sousa, Cruz, Viladrich, y Torregrosa, 2007). Por tanto, el entrenador va a convertirse en uno de los agentes de socialización con mayor influencia sobre los jóvenes deportistas, no sólo desde un paradigma deportivo, sino desde una perspectiva integral que engloba un desarrollo cognitivo, afectivo-social y físico (Morelló, VertBoyer, y Navarro-barquero, 2018; Pulido, Leo, Chamorro, y García-Calvo, 2015).

En este sentido, el ambiente creado por el entrenador puede resultar relevante para desembocar en consecuencias relacionadas con el desarrollo integral de los jóvenes deportistas (Cecchini, Méndez-Jiménez, y Fernández-Rio,
2014). Este entorno, caracterizado por la conducta verbal que adopte el entrenador y por la idiosincrasia de su forma de entrenar, también denominado "estilo interpersonal" (Balaguer et al., 2012), puede determinar el grado de satisfacción o frustración de las necesidades psicológicas básicas de los deportistas y, a su vez, el nivel de autodeterminación que explica el motivo de práctica que lleva al sujeto al desempeño de una actividad (Deci y Ryan, 1985). La Teoría de la Autodeterminación (Deci y Ryan, 1985) proponer diferentes tipos de regulación motivacional, que oscilan desde regulaciones implícitas en la idiosincrasia, voluntariedad y personalidad del sujeto que lo practica (motivación intrínseca), hasta las definidas por ser ajenas a la persona (motivación extrínseca), hasta razones de práctica en las que la persona no encuentra sentido a lo que realiza, ni sabe muy bien el porqué de seguir dedicando tiempo a la actividad (desmotivación).

Nutriendo a la Teoría de la Autodeterminación, Deci y Ryan (2000) 
proponen la existencia de tres necesidades psicológicas universales, innatas y esenciales que van a determinar el grado de autodeterminación de las personas hacia una actividad. Concretamente, estas tres necesidades psicológicas básicas se denominan, necesidad de autonomía, definida como la posibilidad de elección que tiene una persona para llevar a cabo su labor, sintiéndose propietario de sus actos y de guiar de forma deliberada su comportamiento; la necesidad de competencia, que se caracteriza por la percepción de eficacia que tiene una persona cuando tiene que realizar una actividad; $y$, por último, la necesidad de relación, que se define como la sensación de integración que tiene un individuo dentro de un colectivo (Deci y Ryan, 2000).

Para favorecer que estas necesidades estén cubiertas, el entrenador puede mostrar un estilo interpersonal de apoyo, estableciendo estrategias para tener en cuenta el carácter innato de los jugadores, fomentando su participación y facilitando sus interacciones con el grupo o, por el contrario, puede estar más decantado hacia un estilo controlador en el que el entrenador sea quien tome todas las decisiones de forma unilateral, considerando la práctica deportiva como una herramienta meramente competitiva y no de aprendizaje para el jugador. En consecuencia, gran parte de la influencia que el entrenador ejerce sobre el deportista estará condicionada por la forma en la que transmita la información (Fraile, De Diego, Vallejo, y Boada, 2011).

En este sentido, algunas de las estrategias para apoyar la autonomía de los jugadores estarían caracterizadas por intervenciones adaptadas a las circunstancias, desde formas más directivas a formas más democráticas (Adie, Duda, y Ntoumanis, 2012), con el diseño de tareas abiertas para que el jugador pudiese elegir en función de la situación que se le presente. Para apoyar la competencia, algunas de las estrategias que podría utilizar el entrenador sería explicar el objetivo de cada tarea, para qué se realiza y porqué, para que los jugadores entendiesen lo que hacen, adaptar las actividades al nivel de los jugadores para que éstas supongan un reto alcanzable encaminado a la superación personal, aporte de un feedback positivo e individualizado, pautas de actuación definidas... Como estrategias para el apoyo a las relaciones sociales, el entrenador apostaría por la transmisión de valores y principios positivos como el respeto, la dignidad, la cooperación y la colaboración, dirigiría las interacciones hacia aspectos significativos de los jugadores, creando un ambiente de cercanía con sus jugadores siendo receptivo a sus sentimientos y emociones (Haerens et al., 2013), ofreciendo a todos los jugadores las mismas posibilidades de intervención, haciendo que se sientan integrados y reconocidos, e intentaría explicar las situaciones que puedan crear conflicto, siendo lo más objetivo posible (García-Calvo, Sánchez-Oliva, Leo, Amado, y Pulido, 2015).

Por el contrario, un entrenador podría adoptar un estilo interpersonal controlador a la autonomía de los jugadores, utilizando una comunicación autoritaria para imponer una forma preconcebida y determinada de pensar y actuar independientemente de la situación (Bartholomew, Ntoumanis, y ThøgersenNtoumani, 2009), manifestando un excesivo control personal, presión y vigilancia durante la acción de los deportistas, con un uso frecuente de comportamientos instructivos (Tessier et al, 2013) sin tener en cuenta la opinión de los jugadores en el diseño y organización de las tareas (Castillo, González, Fabra, Mercé, y Balaguer, 2012). Se caracterizaría por estrategias que controlan la competencia de los jugadores, aportando un feedback crítico en público, con comparación normativa y externamente referenciado respecto a la acción de sus compañeros, sin tener en cuenta el ritmo individualizado de aprendizaje para estimular la superación personal y fomenten el progreso, ofreciendo información abundante $y$ desestructurada $\mathrm{y}$, centrada en elementos no controlables por el deportista, no existiendo una explicación clara del objetivo que impide saber al 
jugador que es lo que tiene que hacer en cada situación (Van den Berghe et al., 2013). En cuanto a comportamientos controladores de relaciones sociales, el entrenador no mostraría interés por la dimensión socio-afectiva del grupo y priorizaría los temas deportivos sobre otros aspectos significativos para los jóvenes, creando un entorno de trabajo competitivo orientado a la superación de los demás mediante el uso de recompensas tangibles que disminuirían los sentimientos de colaboración y cooperación, provocando situaciones desiguales de participación (Galván, López-Walle, PérezGarcía, Tristán, y Medina-Rodríguez, 2013).

Para ello, es importante que los entrenadores del deporte base dispongan de la formación necesaria que les permita el desarrollo de vínculos interpersonales de colaboración efectiva en el desarrollo de tareas y habilidades (Pulido, Sánchez-Oliva, Sánchez-Miguel, Leo, y García Calvo, 2016), en los que los deportistas que instruyen, interioricen y refuercen conocimientos y habilidades sociales a través del deporte, facilitando e incrementando el desarrollo emotivo y psicosocial (Jowett y Cockerill, 2003).

Sin embargo, son escasos los estudios realizados hasta la fecha acerca del estilo interpersonal que puede adoptar el entrenador teniendo en cuenta su cualificación académica y/o federativa, así como también, hasta nuestro conocimiento, no existen numerosos estudios que hayan llevado a cabo intervenciones con entrenadores en los que se pretenda optimizar algunas de sus habilidades como entrenador. Teniendo en cuenta la formación del entrenador, Leo, García-Calvo, Sánchez-Miguel, SánchezOliva y Gómez (2010) observaron que los entrenadores con formación universitaria aportaban más información de tipo psicológico y feedback de refuerzo que los entrenadores sin formación. En otro estudio acerca de la presentación de las tareas en base a la formación académica de un grupo de entrenadores de fútbol (Mesquita, Farias, Rosado, Pereira, y Moreno, 2008), se obtuvo como resultado que los entrenadores licenciados en Educación Física y
Deporte se distinguen significativamente de los no licenciados al emplear más tareas de perfeccionamiento e indicar más a los jugadores los criterios de realización de las tareas. Estos resultados son similares a los obtenidos por Feu, Ibáñez y Gozalo (2010), destacando que los entrenadores con un mayor nivel de formación académica $\mathrm{y} / \mathrm{o}$ federativa mostraban comportamientos más deseables como la orientación al trabajo en equipo. Estos resultados son corroborados por Sánchez-Oliva, SánchezMiguel, Amado, Leo y García-Calvo (2010), que destacan que los entrenadores cualificados dan información más breve y un feedback más de tipo afectivo y prescriptivo.

Es por esto que, tras la revisión de la literatura científica, encontramos antecedentes en el diseño y aplicación de programas de formación para entrenadores, de entre los que destacan el programa Coach Efectiviness Training (Smith, Smoll, y Curtis, 1979), pionero y precursor de otros programas como el Coach Analysis Instrument (Kenneth, More, y Franks, 1996), o el Mastery Aproach to Coaching (Smith, Smoll, y Cumming, 2007). Resultado del perfeccionamiento de los dos programas anteriores y en el ámbito específico del fútbol, surge el Programa de Asesoramiento Personalizado a Entrenadores (Sousa, Cruz, Torregrosa, Vilches, y Viladrich, 2006). Bajo esta misma tendencia, nació el Promoting Adolescent Physical Activity (Duda et al., 2013).

Tras analizar los estudios publicados hasta la fecha, se observan que son escasas las investigaciones que han examinado los efectos de programas de formación para entrenadores, pero son menos aun los que se basan en el desarrollo de estrategias metodológicas para optimizar el ambiente que genera el entrenador durante el desarrollo de su función (Pulido et al., 2015). Por tanto, a través de este estudio se pretenden comprobar los efectos de un programa de formación para entrenadores sin cualificación federativa y académica relacionada con la actividad física y el deporte basado en estrategias metodológicas y motivacionales con el propósito 
de optimizar el comportamiento y conducta verbal que utilizan los entrenadores durante el desempeño de su labor con jóvenes deportistas. La hipótesis que planteamos es que los entrenadores que reciben el programa de formación, modificarán su estilo interpersonal, incrementando sus comportamientos de apoyo y reduciendo sus comportamientos de control.

\section{MATERIAL Y MÉTODOS}

\section{Participantes}

La muestra del estudio estaba constituida por ocho entrenadores de género masculino de las categorías infantil y cadete de una liga regional. Los entrenadores se repartieron equitativamente en grupo control $(n=4)$ y en grupo experimental $(n=4)$, con experiencia dirigiendo a equipos entre 1 y 6 años $(M=3,00 ; D T=2,44)$ y entre 3 y 10 años $(M=6,25 ; D T=2,87)$, respectivamente $\mathrm{y}$, con una formación académica de Educación General Básica y/o Educación Secundaria Obligatoria.

La elección de los entrenadores se realizó a través de un muestreo intencional, estableciendo como requisito no tener ninguna formación superior relacionada con el deporte, ni de tipo académico (universitaria $\mathrm{y} / \mathrm{o}$ técnica), ni federativo. Una vez cumplido este filtro, se realizó una distribución aleatoria por pares para establecer una repartición normal de las categorías a las que entrenaban y de los años de experiencia (dos entrenadores de categoría infantil y dos entrenadores de categoría cadete al grupo control y otros tantos al experimental).

\section{Instrumentos}

Para la observación del estilo interpersonal del entrenador se utilizó un instrumento de observación denominado Coach Interpersonal Style Observational System (CISOS; Pulido, Sánchez-Oliva, Silva, y García-Calvo, en revisión). Dicha herramienta clasifica las intervenciones del entrenador en siete macrodimensiones (tabla 1) en base a aspectos metodológicos: 1) Información Inicial (el entrenador explica la tarea a realizar), 2)
Reconducción Durante el Proceso (el entrenador aporta información de recuerdo o correcciones durante la tarea, en partidos se denominaría Reconducción Durante el Juego), 3) Clima Ego/Tarea (indicaciones que orientan hacia el resultado o el aprendizaje), 4) Instrucción Técnico-Táctica General (indicaciones verbales antes de que se produzca una acción, pueden ser colectivas o individuales), 5) Feedback (correcciones o refuerzos tras una acción, pueden ser colectivas o individuales) 6) Clima Social (ambiente afectivo-emocional creado por el entrenador, también puede ser colectivo o individual), y 7) Otros (interacción del entrenador que no tienen nada que ver con el desarrollo deportivo ni es influyente para los jugadores). Además, cada categoría es valorada en tres dimensiones (grados de apertura o plasticidad), dependiendo del estilo mostrado por el entrenador (Anguera y Hernández-Mendo, 2014): apoyo (el entrenador utiliza una comunicación para explicar, dar instrucciones deportivas, corregir..., con el objetivo que se produzca, de manera consciente o no, un aprendizaje significativo en los deportistas); neutro (la interacción comunicativa entrenador-deportista en cada una de sus macrodimensiones es asertiva y no converge en un comportamiento verbal de apoyo o control); y control (la relación entrenadorjugador es totalmente jerárquica y el entrenador lo manifiesta mediante conductas imperativas, dando importancia al resultado y al cumplimiento de las normas por él establecidas).

\section{Procedimiento}

En primer lugar, se pidió el consentimiento a la Comisión Ética de la Universidad. Seguidamente, los investigadores se pusieron en contacto con los dirigentes de los clubes y entrenadores de los equipos informándoles sobre el estudio. Se les explicó el objetivo del mismo y la metodología a seguir, indicando a los entrenadores que se trataba de un estudio observacional. Además, todos los jugadores deberían entregar a sus padres o tutores legales un consentimiento informado en el que autorizaban la grabación de audio y vídeo durante 
los entrenamientos. Todos los sujetos fueron informados de que su participación era voluntaria y que los archivos multimedia serían tratados de forma confidencial con un uso exclusivo para investigación.

A continuación, se constituyó un grupo de investigadores que fueron los responsables de la grabación del audio y vídeo de los entrenamientos para llevar a cabo las dos tandas de medición medidas. Inicialmente, se grabó un entrenamiento de cada entrenador para la realización de una prueba piloto. Posteriormente, se grabaron dos entrenamientos de cada entrenador en la fase previa al programa e igualmente se filmaron dos entrenamientos de cada entrenador tras la intervención. La duración media de la grabación de cada entrenamiento fue de 1 hora y 30 minutos.

\section{Programa de intervención}

Tras la obtención de todos los datos (filmación y edición de los vídeos y archivos de audio), se llevó a cabo un programa de entrenamiento para los observadores. Tras el proceso de formación, los codificadores deberían obtener un índice de confiabilidad por encima de 0,90 (Brewer y Jones, 2002). La fiabilidad inter-observadores se basó en la concordancia del estándar mínimo de codificación y el coeficiente de correlación intraclase (ICC) resultante por una prueba realizada conjuntamente por el investigador principal ('gold standard'). El ICC se interpreta como un valor pobre por debajo de 0,50, moderado si está entre 0,50 y 0,75 , y adecuado si es superior a 0,75 (Portney y Watkins, 2009). De esta forma, cuatro observadores (todos hombres) especialistas en Psicología y Fútbol e independientes al diseño y desarrollo del sistema de observación, fueron entrenados y evaluados de acuerdo a criterios de fiabilidad, mediante la visualización de cinco fragmentos secuenciales de 15-minutos de duración (rango de acuerdo en las pruebas finales de codificación $=79-89 \%$ de acuerdo, rango Kappa $=.63-.69 ; M$ ICC $=.96$ ) durante el período de dos meses. Los re-test de fiabilidad inter-observadores fueron realizados tras dos meses desde el comienzo de entrenamiento de los observadores y el estándar mínimo del $75 \%$ de acuerdo se había mantenido (rango $=84-94 \%$ de acuerdo, rango Kappa $=.66$ - .93; M ICC = .97).

Para la clasificación de las técnicas de registro, se siguió un criterio taxonómico basado en los comportamientos de los entrenadores según un registro de eventos (Anguera, Blanco-Villaseñor, Hernández-Mendo, y Losada, 2011) en cuanto a su repetitividad, en el que cada vez que se producía un comportamiento verbal, se clasificaba y catalogaba según su dimensión. La duración de cada análisis observacional osciló entre las tres y cuatro horas de media. Inicialmente, los datos fueron recogidos en una hoja del instrumento de observación, permitiendo la codificación y simplificación de los datos. Sobre esta hoja, a cada unidad de análisis se le asignaba un grado de apertura con un valor en función del estilo que mostraba el entrenador en la categoría correspondiente, quedando registradas las frecuencias de las puntuaciones de cada núcleo categorial. 
Pulido, J. J. (1), Merino, A. (2), Sánchez-Oliva, D. (3), Tapia, M. A. (4), y García-Calvo, T. (5)

Tabla 1. Macrodimensiones, dimensiones y ejemplos de cada una de las unidades de análisis del instrumento de observación.

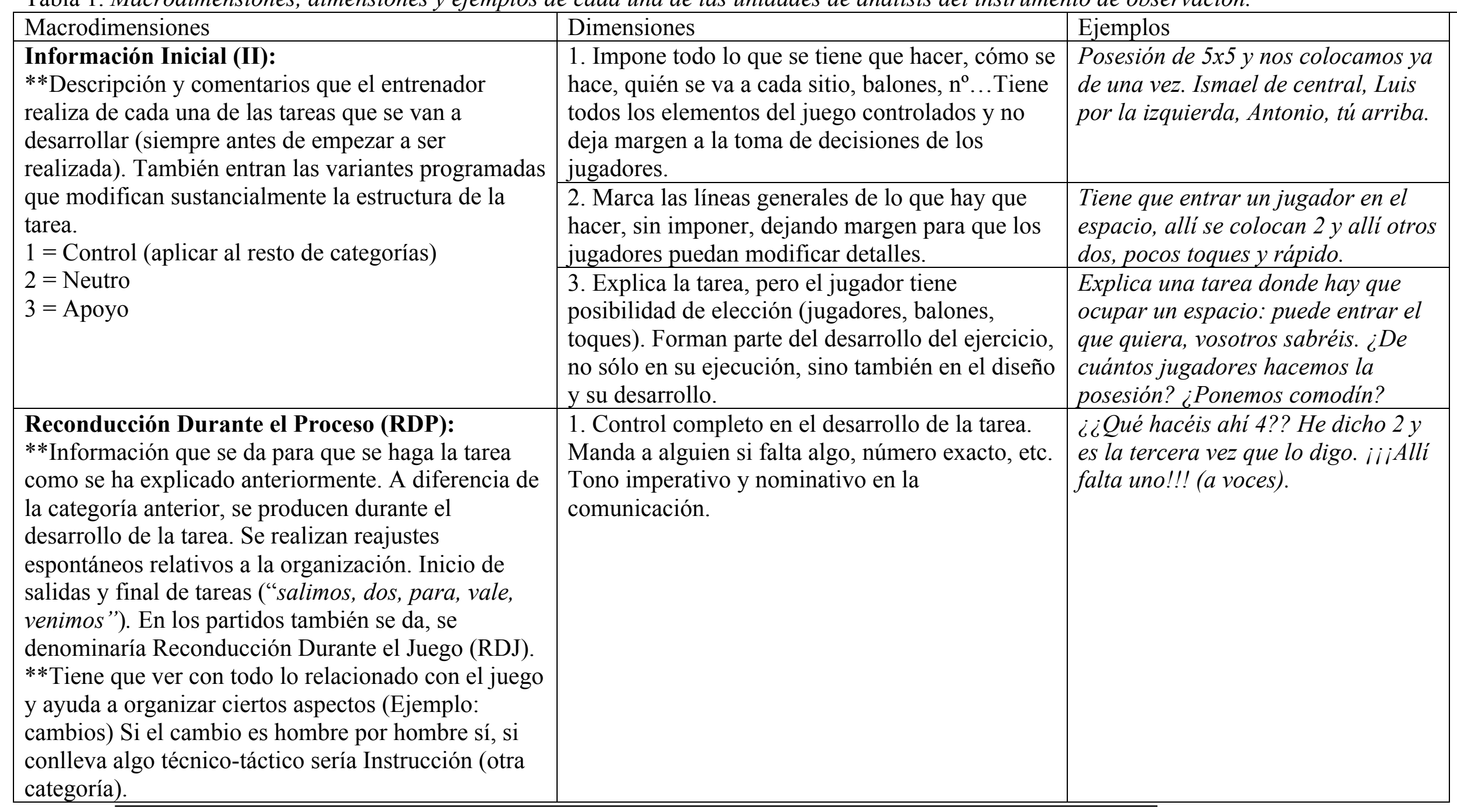




\section{Efectos de un programa de intervención sobre el estilo interpersonal de entrenadores de fútbol base}

Tabla 1. Macrodimensiones, dimensiones y ejemplos de cada una de las unidades de análisis del instrumento de observación. (cont.)

\begin{tabular}{|c|c|c|}
\hline \multirow[t]{2}{*}{$\begin{array}{l}\text { Reconducción Durante el Proceso (RDP) } \\
\text { (cont.): }\end{array}$} & $\begin{array}{l}\text { 2. Se preocupa porque el ejercicio se desarrolle } \\
\text { como ha explicado, pero ni controla ni apoya } \\
\text { con sus intervenciones. }\end{array}$ & $\begin{array}{l}\text { Poneros } 2 \text { allí, tiene que haber } 2 \text {, } \\
\text { venga. Dije que tenía que haber } 5 \\
\text { (sólo indica/corrige, tampoco apoya, } \\
\text { no cabreo). }\end{array}$ \\
\hline & $\begin{array}{l}\text { 3. Tiene en cuenta las opiniones de los } \\
\text { jugadores durante el funcionamiento de las } \\
\text { tareas. Iniciativa del entrenador para que los } \\
\text { jugadores participen en la RP (formula } \\
\text { preguntas). }\end{array}$ & $\begin{array}{l}\text { Dos que quieran que se pongan allí. } \\
\text { Ok, un balón más (tras una pregunta } \\
\text { de un jugador). ¿No creéis que falta } \\
\text { algún jugador allí para que estemos } \\
\text { equilibrados? (iniciativa del } \\
\text { entrenador). }\end{array}$ \\
\hline \multirow{3}{*}{$\begin{array}{l}\text { Clima Ego/Tarea (CET): } \\
* * \text { Referencia hacia los refuerzos que incitan a } \\
\text { la competitividad o la ejecución. Si el } \\
\text { entrenador fomenta la competitividad durante } \\
\text { la explicación o se centra en el proceso de } \\
\text { aprendizaje (adquisición de habilidades), } \\
\text { ejemplo: quien marque, gana (se centra en el } \\
\text { resultado y no en el proceso) o valorar el } \\
\text { aprendizaje, no el marcador (se centra en la } \\
\text { tarea y no en el resultado (ejemplo: si no sale } \\
\text { es normal, estamos aprendiendo). }\end{array}$} & $\begin{array}{l}\text { 1. Focalización de la tarea en el resultado y } \\
\text { comparación entre compañeros y/o rivales. } \\
\text { Fomento de la competitividad. }\end{array}$ & $\begin{array}{l}\text { Quien pierda da } 10 \text { vueltas. El último } \\
\text { en llegar ha sido Juan. }\end{array}$ \\
\hline & $\begin{array}{l}\text { 2. No incita ni a la competición ni a la tarea, } \\
\text { pero nombra ambas cosas (no lo deja claro). } \\
\text { Comentarios contradictorios. }\end{array}$ & $\begin{array}{l}\text { Quién más robos realice, gana, pero } \\
\text { no olvidéis hacerlo bien (es un } \\
\text { mensaje ambiguo, no deja claro la } \\
\text { orientación). }\end{array}$ \\
\hline & $\begin{array}{l}\text { 3. Focaliza la atención en el aprendizaje y no en } \\
\text { el resultado. }\end{array}$ & $\begin{array}{l}\text { Es normal que os cueste al principio } \\
\text { pero prefiero que aprendamos, } \\
\text { aunque nos cueste algún gol (salida } \\
\text { de balón). }\end{array}$ \\
\hline
\end{tabular}


Pulido, J. J. (1), Merino, A. (2), Sánchez-Oliva, D. (3), Tapia, M. A. (4), y García-Calvo, T. (5)

Tabla 1. Macrodimensiones, dimensiones y ejemplos de cada una de las unidades de análisis del instrumento de observación. (cont.)

\begin{tabular}{|c|c|c|}
\hline \multirow{3}{*}{$\begin{array}{l}\text { Instrucción técnico-táctica General } \\
\text { (IGE): } \\
\text { **Son comportamientos y conductas } \\
\text { verbales que se producen } \\
\text { espontáneamente (no existe acciones } \\
\text { previas) y están relacionadas con el } \\
\text { desarrollo del juego. No tienen que ver } \\
\text { con un error-consecuencia. Se producen } \\
\text { siempre previamente a la acción, sino } \\
\text { sería un feedback. También van } \\
\text { asociados a una explicación sobre cómo } \\
\text { hacer algo (ej.: planteamiento para el } \\
\text { próximo partido). El "ánimo" en } \\
\text { cuestiones técnico-tácticas (TT): } \\
\text { "estamos dormidos, intensidad, vamos } \\
\text { fuerte ahora". } \\
\text { COLECTIVO (IGEC): a todo el grupo o } \\
\text { varios jugadores. } \\
\text { INDIVIDUAL (IGEI): personalizado. }\end{array}$} & \multicolumn{2}{|c|}{$\begin{array}{l}\text { Instrucción técnico-táctica General } \\
\text { (IGE): } \\
\text { **Son comportamientos y conductas }\end{array}$} \\
\hline & $\begin{array}{l}\text { 2. Retransmite de forma aséptica cosas del juego } \\
\text { pero no ofrece ni control ni apoyo. }\end{array}$ & $\begin{array}{l}\text { Vigilancias, marcajes, fuertes, } \\
\text { tenemos que despejar, etc. }\end{array}$ \\
\hline & $\begin{array}{l}\text { 3. Da indicaciones de lo que hay que hacer, pero } \\
\text { ofrece libertad para que se produzcan decisiones } \\
\text { por parte de los jugadores. Da confianza de lo TT } \\
\text { antes de que se produzca y explica la importancia } \\
\text { de hacerlo de esa manera. }\end{array}$ & $\begin{array}{l}\text { Quiero que la gente hable } \\
\text { (ofrece comunicación entre } \\
\text { ellos). Uno de los dos } \\
\text { centrales sube, Uno de los } \\
\text { extremos más abiertos, } \\
\text { ¿Quién se ofrece para marcar } \\
\text { al 9? ¿Quién quiere sacar la } \\
\text { falta? Quiero que toquemos } \\
\text { rápido para que no les dé } \\
\text { tiempo a bascular (explica el } \\
\text { por qué, la razón de hacerlo). }\end{array}$ \\
\hline \multirow{2}{*}{$\begin{array}{l}\text { Feedback (F): } \\
\text { **Corrección ante acierto o error } \\
\text { (positivas y/o negativas y/o neutras). } \\
\text { Reflexiones que hace el entrenador tras } \\
\text { las acciones técnico-tácticas de los } \\
\text { jugadores, dando una información, } \\
\text { opinión, }\end{array}$} & $\begin{array}{l}\text { 1. Desprestigio del entrenador sobre la/s } \\
\text { acción/es del/los jugador/es (punitiva). }\end{array}$ & $\begin{array}{l}\text { Vaya tiro Lolo, vaya tela. } \\
\text { ¿Pero qué hacéis ahora? } \\
\text { Fatal. ¡Anda que...vaya dia } \\
\text { lleváis hoy! }\end{array}$ \\
\hline & & \\
\hline
\end{tabular}

opinión, 
Efectos de un programa de intervención sobre el estilo interpersonal de entrenadores de fútbol base Tabla 1. Macrodimensiones, dimensiones y ejemplos de cada una de las unidades de análisis del instrumento de observación.

\begin{tabular}{|c|c|c|}
\hline \multirow{2}{*}{$\begin{array}{l}\text { Feedback (F) (cont.): } \\
\text { posicionamiento de lo que ha hecho y } \\
\text { cómo se ha hecho. La diferencia de la } \\
\text { anterior es que hay una acción } \\
\text { (jugador) - consecuencia (jugador). } \\
\text { COLECTIVO (FC): a todo el grupo o } \\
\text { varios jugadores. } \\
\text { INDIVIDUAL (FI): personalizado. }\end{array}$} & $\begin{array}{l}\text { 2. Se refiere a la simple corrección de la acción sin } \\
\text { ningún porqué razonado (más fuerte, más flojo, más } \\
\text { adelante) (siempre tras una acción). }\end{array}$ & $\begin{array}{l}\text { Cuerpo encima, has puesto el } \\
\text { cuerpo hacia atrás. Control } \\
\text { orientado, el control no estaba } \\
\text { orientado. iNo tan arriba! }\end{array}$ \\
\hline & $\begin{array}{l}\text { 3. Refuerzo positivo, reconocimiento del progreso y } \\
\text { confianza del entrenador en la ejecución. Reflexión } \\
\text { de lo que es mejor como forma de aprendizaje. } \\
\text { También, feedback interrogativo que implica } \\
\text { cognitivamente al deportista. }\end{array}$ & $\begin{array}{l}\text { Aunque nos ha costado un gol } \\
\text { habéis hecho muy bien en estar } \\
\text { adelantados, porque en muchos } \\
\text { partidos cortamos muchos } \\
\text { contra-ataques. Si es zurdo, } \\
\text { ¿Por qué lado crees que es más } \\
\text { fácil desbordarle? }\end{array}$ \\
\hline \multirow{2}{*}{$\begin{array}{l}\text { Clima Social (CS): } \\
\text { ** Generación del clima que genera el } \\
\text { entrenador durante los entrenamientos } \\
\text { y los partidos con connotación } \\
\text { afectivo-emocional. También se } \\
\text { refiere a la cercanía, respeto, disciplina } \\
\text { y esfuerzo por la integración que } \\
\text { transmite el entrenador sobre sus } \\
\text { jugadores. También entraría el ánimo } \\
\text { general y las posibilidades de } \\
\text { intervenir. Puede derivarse de otra } \\
\text { categoría que también incide en el } \\
\text { clima general del equipo o relación } \\
\text { jugador/entrenador. }\end{array}$} & $\begin{array}{l}\text { 1. Falta de respeto grave del entrenador hacia } \\
\text { un/varios jugador/es y/o despreocupación por la } \\
\text { integración y ambiente positivo del equipo. Castigos. }\end{array}$ & $\begin{array}{l}\text { Si le das (patada), mejor, etc. } \\
\text { Palabras malsonantes y } \\
\text { ambiente tenso. Insultos con } \\
\text { menosprecio a jugadores. }\end{array}$ \\
\hline & $\begin{array}{l}\text { 2. Aspectos relacionados con el ambiente de grupo } \\
\text { que no tienen una inclinación positiva ni negativa. }\end{array}$ & $\begin{array}{l}\text { El entrenamiento es a las } 8 \text { (ha } \\
\text { llegado tarde). ;Va! iVenga! (sin } \\
\text { significar nada, muletilla). }\end{array}$ \\
\hline
\end{tabular}

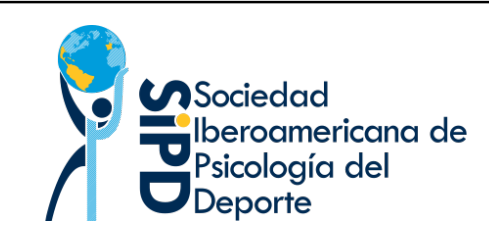


Pulido, J. J. (1), Merino, A. (2), Sánchez-Oliva, D. (3), Tapia, M. A. (4), y García-Calvo, T. (5)

Tabla 1. Macrodimensiones, dimensiones y ejemplos de cada una de las unidades de análisis del instrumento de observación.

\begin{tabular}{|c|c|c|}
\hline $\begin{array}{l}\text { Clima Social (CS) (cont.): } \\
\text { COLECTIVO (CSC): a todo el } \\
\text { grupo o varios jugadores. } \\
\text { INDIVIDUAL (CSI): } \\
\text { personalizado. }\end{array}$ & $\begin{array}{l}\text { 3. Referencia y preocupación del entrenador } \\
\text { como modelo a seguir y buen ambiente de } \\
\text { grupo, cooperación, transmisión de respeto, } \\
\text { disciplina, educación e integración. También, } \\
\text { ánimo general. }\end{array}$ & $\begin{array}{l}\text { No se protesta al árbitro. Vamos a llevarnos } \\
\text { bien. Arriba equipo. ¿Y esas notas Carlos? } \\
\text { ¡Parad!, se ha hecho daño Chema... Cuando } \\
\text { falla el compañero, debemos animarle. }\end{array}$ \\
\hline \multirow{3}{*}{$\begin{array}{l}\text { Otros (O): } \\
* * \text { Cosas irrelevantes que no } \\
\text { afectan a los jugadores y al } \\
\text { desarrollo del entrenamiento y } \\
\text { partidos. Ejemplo: cuando el } \\
\text { entrenador habla en privado con } \\
\text { padres, entrenadores, árbitros, } \\
\text { etc. Si lo hace delante de sus } \\
\text { jugadores, no se encuadraría en } \\
\text { estas categorías. }\end{array}$} & $\begin{array}{l}\text { 1. Cuando habla con un } \\
\text { árbitro/padre/entrenador. Podría afectar } \\
\text { indirectamente los jugadores: discusión fuerte. }\end{array}$ & $\begin{array}{l}\text { Insultos a agente de socialización } \\
\text { (entrenadores, árbitros, padres, otros } \\
\text { jugadores) fuera del alcance perceptivo de sus } \\
\text { jugadores. }\end{array}$ \\
\hline & $\begin{array}{l}\text { 2. Cuando habla con un } \\
\text { árbitro/padre/entrenador y no afecta, ni directa, } \\
\text { ni positiva ni negativamente a los jugadores: } \\
\text { charla normal. }\end{array}$ & $\begin{array}{l}\text { Habla con un entrenador rival de manera en } \\
\text { un tono neutro al inicio o final del partido. }\end{array}$ \\
\hline & $\begin{array}{l}\text { 3. Cosas que no ocurren entre entrenador- } \\
\text { jugador y podrían a llegar a afectar positiva e } \\
\text { indirectamente al equipo. }\end{array}$ & $\begin{array}{l}\text { Habla con un árbitro/padre/entrenador fuera } \\
\text { del alcance de los jugadores: } \\
\text { recomendaciones, preocupaciones en tono } \\
\text { amigable y distendido. }\end{array}$ \\
\hline
\end{tabular}


La intervención con los entrenadores consistió en instruir al grupo experimental $(n=4)$ mediante un programa de formación impartido por especialistas en Psicología del Deporte y Fútbol. Dicho programa (Pulido, Sánchez-Oliva, Leo, Matos, y García-Calvo, 2017) estaba basado en el fomento de estrategias metodológicas y motivacionales. La duración total de la formación fue de 12 horas, divididas en tres sesiones (cuatro horas cada una). El programa de formación estaba compuesto por dos partes: 1) Contextualización y marco teórico centrado en la Teoría de la Autodeterminación (Deci y Ryan, 1985) y su relación con la práctica deportiva, importancia de las necesidades psicológicas básicas de autonomía, competencia y relaciones sociales (Deci y Ryan, 2000), influencia de los agentes sociales (entrenador) sobre los procesos motivaciones y estudios que han demostrado la importancia de programas de formación basados en la teoría en diferentes ámbitos de actuación: académico, laboral, deportivo... 2) Estrategias metodológicas y motivacionales para optimizar las necesidades psicológicas básicas de los deportistas. Como ejemplo, para fomentar la necesidad de competencia se incidía en la importancia de adaptación del aprendizaje, explicaciones basadas en el progreso, la importancia del feedback centrados en la individualización, el planteamiento de retos alcanzables partiendo de un equilibrio entre dificultad/habilidad...

Tras la aplicación del programa de formación y antes de la última observación, se dejó un período de ocho semanas para la asimilación y puesta en práctica por parte de los entrenadores de los contenidos impartidos durante el proceso de intervención.

\section{Análisis estadístico}

Para el tratamiento y análisis de los datos se utilizó el programa SPSS 22.0. De este modo, las conductas observadas a nivel categórico, fueron cuantificadas en forma de frecuencias/indicadores (media y desviación típica). Seguidamente, fueron estimados los estadísticos descriptivos de todas las variables dependientes correspondientes a cada una de las categorías del instrumento, en el pre-test y en el post-test. A continuación, para analizar las diferencias inter-grupo entre el pretest y el post-test, se realizó un análisis de varianza de medidas repetidas (MANOVA) de 2 (grupo: control y experimental) x 2 (medidas: pretest y post-test). En el mismo procedimiento se comprobaron las diferencias del grupo experimental entre la toma pre-test y la toma posttest.

\section{RESULTADOS}

A continuación, se muestran los resultados descriptivos obtenidos en todas las categorías registradas en el estudio. En la tabla 2, se observan los valores de las dos medidas realizadas (pre-test y pos-test) y de los dos grupos (control y experimental), en el que los valores iniciales correspondientes al estilo interpersonal de los entrenadores del grupo control y experimental en todas las categorías son similares. Además, se exponen los resultados obtenidos del análisis de la varianza de medidas repetidas (MANOVA), que indica que existen cambios en el estilo interpersonal de los entrenadores del grupo experimental al final de la intervención, a pesar de que no existen diferencias significativas en la evolución de ambos grupos.

Posteriormente, al considerar que el análisis inferencial de los datos no reflejaba resultados concluyentes en relación a la consecución de los objetivos planteados, se decidió llevar a cabo un análisis más descriptivo de las variables, considerando el porcentaje de intervenciones del entrenador que son de apoyo o controladoras en el conjunto de todas las categorías. Se puede observar que existe una diferente evolución entre el grupo control y el experimental en el valor medio de las conductas de control de todas las categorías (MC) de los entrenadores del grupo control y experimental.

Tabla 2. Estadísticos descriptivos y efectos del programa en la interacción.

\begin{tabular}{cc|cc|c}
\hline \multicolumn{2}{c}{ Control } & \multicolumn{2}{c}{ Experimental } & Grupo*Tiempo \\
\hline Pre & Post & Pre & Post & \\
$\mathrm{M}(D T)$ & $\mathrm{M}(D T)$ & $\mathrm{M}(D T)$ & $\mathrm{M}(D T)$ & $p$ \\
\hline
\end{tabular}


Pulido, J. J. (1), Merino, A. (2), Sánchez-Oliva, D. (3), Tapia, M. A. (4), y García-Calvo, T. (5)

\begin{tabular}{ccc|cc|c}
\hline II & $1,75 \pm$ & $1,93 \pm$ & $1,66 \pm$ & $2,01 \pm$ & 0,085 \\
& $(0,15)$ & $(0,25)$ & $(0,13)$ & $(0,13)$ & \\
RDP & $1,82 \pm$ & $1,89 \pm$ & $1,83 \pm$ & $1,98 \pm$ & 0,472 \\
& $(0,11)$ & $(0,14)$ & $(0,14)$ & $(0,12)$ & \\
CET & $1,45 \pm$ & $1,24 \pm$ & $1,28 \pm$ & $1,15 \pm$ & 0,809 \\
& $(0,49)$ & $(0,18)$ & $(0,28)$ & $(0,23)$ & \\
IGEC & $1,85 \pm$ & $2,00 \pm$ & $1,88 \pm$ & $2,02 \pm$ & 0,908 \\
& $(0,07)$ & $(0,11)$ & $(0,14)$ & $(0,14)$ & \\
IGEI & $1,38 \pm$ & $1,43 \pm$ & $1,39 \pm$ & $1,54 \pm$ & 0,691 \\
& $(0,30)$ & $(0,16)$ & $(0,06)$ & $(0,20)$ & \\
FC & $2,33 \pm$ & $2,08 \pm$ & $2,46 \pm$ & $2,35 \pm$ & 0,508 \\
& $(0,19)$ & $(0,23)$ & $(0,10)$ & $(0,92)$ & \\
FI & $2,27 \pm$ & $2,11 \pm$ & $2,50 \pm$ & $2,51 \pm$ & 0,423 \\
& $(0,26)$ & $(0,36)$ & $(0,96)$ & $(0,15)$ & \\
CSC & $2,11 \pm$ & $2,49 \pm$ & $2,25 \pm$ & $2,45 \pm$ & 0,614 \\
& $(0,31)$ & $(0,35)$ & $(0,26)$ & $(0,22)$ & \\
CSI & $2,34 \pm$ & $2,24 \pm$ & $2,15 \pm$ & $2,34 \pm$ & 0,387 \\
& $(0,22)$ & $(0,48)$ & $(0,27)$ & $(0,27)$ & \\
O & $2,08 \pm$ & $2,13 \pm+$ & $2,25 \pm$ & $2,11 \pm$ & 0,247 \\
& $(0,14)$ & $(0,18)$ & $(0,07)$ & $(0,09)$ & \\
\hline MC & $1,96 \pm$ & $1,93 \pm$ & $1,95 \pm$ & $2,07 \pm$ & 0,268 \\
& $(0,10)$ & $(0,17)$ & $(0,10)$ & $(0,07)$ & \\
\hline
\end{tabular}

Seguidamente, se muestra la evolución, desde la medida pre-intervención a la posintervención, de la media del porcentaje obtenido en conductas de apoyo (Figura 1) y control (Figura 2) de todas las categorías analizadas (Med\%ContCat) en los grupos control y experimental. Se observa en el grupo de intervención un aumento en la media del porcentaje de las conductas de apoyo en un $2,11 \%$, mientras que en el grupo de control se produce un descenso en un 1,47\%. En cuanto a la media del porcentaje de conductas de control, se observa una reducción en el grupo experimental en un $4,4 \%$, mientras que el grupo control aumenta su media en el porcentaje en un $2,2 \%$.

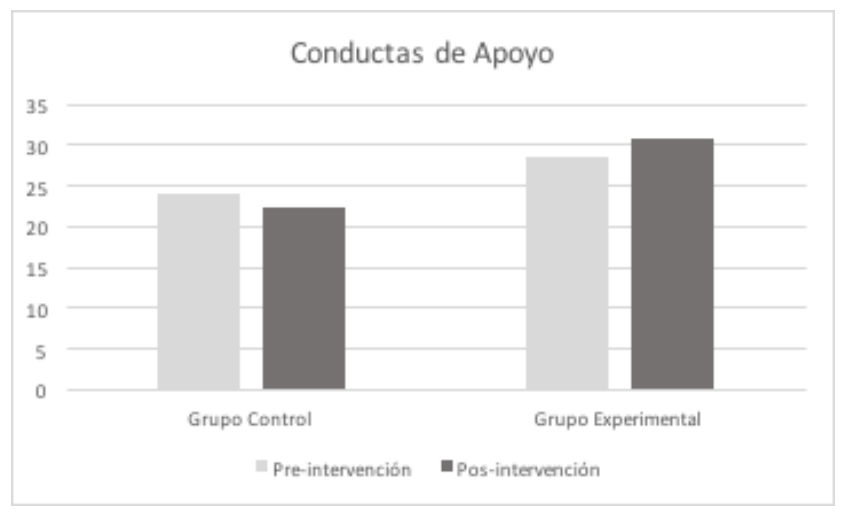

Figura 1. Evolución de la media del porcentaje de comportamientos de apoyo de todas las categorías en los grupos control y experimental.

Figura 2. Evolución de la media del porcentaje de comportamientos de control de todas las categorías en los grupos control y experimental.

Por último, se ilustra (Figura 3) la evolución de la media de los porcentajes de apoyo $\mathrm{y}$ control de todas las categorías en los entrenadores del grupo experimental. De los cuatro entrenadores del grupo experimental, el entrenador 1 y 2 muestran un aumento de la media del porcentaje de apoyo y disminuyen la media

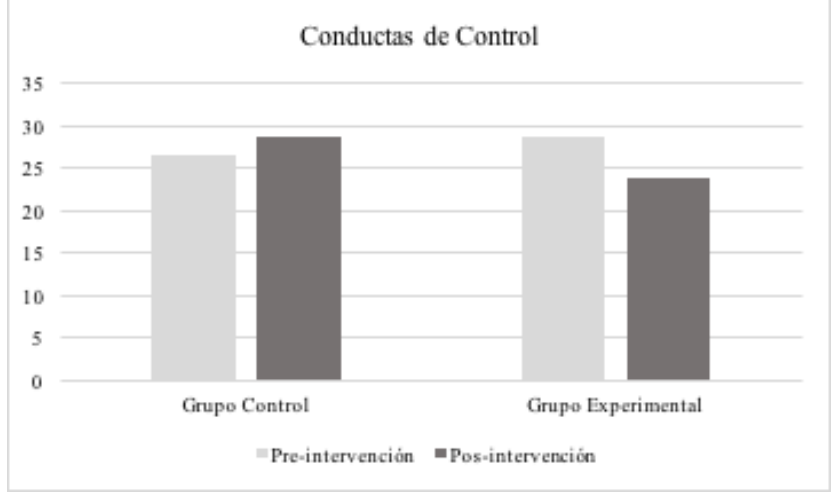

del porcentaje de comportamientos de control. El entrenador 3 disminuye su media del porcentaje de comportamientos de apoyo en un 5,66\% y, la media del porcentaje de control en un $9,15 \%$. El cuarto entrenador reduce su media de porcentaje de apoyo y aumenta ligeramente la media del porcentaje de conductas de control.

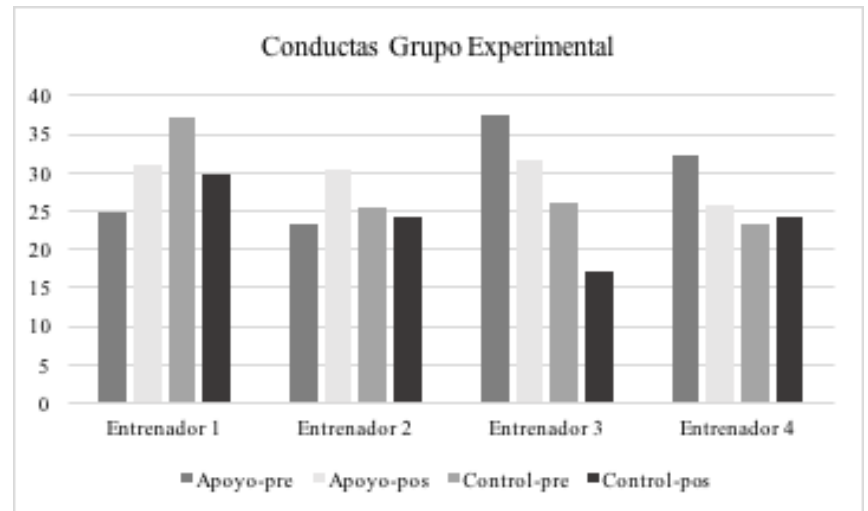


Figura 3. Evolución de la media de los porcentajes apoyo y control de todas las categorías en los entrenadores del grupo experimental.

\section{DISCUSIÓN}

Teniendo en cuenta el objetivo del estudio que era comprobar los efectos de un programa de formación sobre el estilo interpersonal que adoptan los entrenadores durante el desarrollo de su labor, los resultados obtenidos no muestran cambios significativos en el aumento de los porcentajes de conductas verbales de apoyo de los entrenadores del grupo experimental tras la intervención. No obstante, se puede observar una tendencia positiva generalizada del grupo experimental, en el que han disminuido la media del porcentaje de comportamientos de control y un aumento de la media de conductas de apoyo en el conjunto de categorías del instrumento, mientras que en el grupo control se produce un descenso en los comportamientos de apoyo y un aumento en las conductas de control. En este sentido y considerando la hipótesis principal planteada, dos de los cuatro entrenadores del grupo experimental han modificado su comportamiento tras la formación, incrementando las intervenciones de apoyo para cada una de las categorías del instrumento $\mathrm{y}$, reduciendo las intervenciones de control, uno de ellos ha descendidos tantos sus conductas verbales de apoyo como de control y, otro de los entrenadores aumenta sus comportamientos de control y desciende los apoyos.

De esta forma, se un perfil de entrenador que aumenta sus intervenciones verbales de apoyo y reduce las de control. Concretamente, se observa cómo los entrenadores 1 y 2 del grupo experimental (ver gráfico 2 ) reducen su media del porcentaje de control de todas las categorías y aumentan la media de los porcentajes de apoyo. Por otro lado, se da otro perfil de entrenador (entrenador 3) que reduce su media del porcentaje de conductas de apoyo y control en la medida final respecto a la inicial. En cambio, el entrenador 4 muestra un perfil totalmente contrario a los entrenadores 1 y 2 , obteniendo una media más elevada del porcentaje de control y un porcentaje más bajo en el nivel de apoyo en la medida final respecto a la inicial. Relacionado con este tipo de perfil de entrenador variado, un estudio realizado con entrenadores de fútbol base (Cruz, Torregrosa, Sousa, Mora, y Viladrich, 2010) obtuvo como resultado que dos de los entrenadores del grupo de intervención no lograron reducir sus conductas de castigo y aumentar significativamente las conductas de refuerzo. Esto puede ser interpretado como que no lograron incrementar las intervenciones de apoyo y reducir las de control. Una posible explicación a estos hallazgos es que pueden existir variables no controladas en ambos estudios, como podrían ser los niveles autodeterminados de los entrenadores previo al programa de intervención $\mathrm{y}$ otras variables motivacionales, el grado de satisfacción en la labor que desempeña, la predisposición al cambio en su forma de transmitir la información... constructos que podrían incidir en los resultados encontrados tras el programa de formación.

Por otro lado, existen estudios que han desarrollado programas de formación en el que existía una diferencia inicial en la cualificación inicial en los entrenadores participantes. En esta línea, Sousa et al. (2006), tras la evaluación de un programa de asesoramiento individualizado a entrenadores, obtuvieron como resultado al comparar la evolución de cuatro técnicos, que el entrenador que disponía de titulación académica en Educación Física y el nivel II (técnico deportivo de nivel medio) de entrenador en fútbol es el que mostró un mayor aumento de las conductas de apoyo y disminución de las conductas de castigo. Por su parte, el entrenador que no poseía ningún tipo de formación, ni académica ni federativa, es el único que no aumentó sus conductas de apoyo tras la intervención. Esto nos hace pensar que, posiblemente, los entrenadores con menor nivel de cualificación o sin ella, son menos propensos o encuentran mayor dificultad de aplicar los conocimientos adquiridos a su labor como 
entrenador, en este caso, a introducir cambios en su estilo interpersonal.

En este sentido, un estudio desarrollado sobre un programa de intervención individualizado con entrenadores de fútbol (Soriano, Ramis, Cruz, y Sousa, 2014), avala los resultados del presente estudio, puesto que de los tres entrenadores que participaron en el estudio, el entrenador que no disponía de titulación ni académica ni federativa, disminuyó sus conductas de castigo solamente en un $5,1 \%$ frente al $17,47 \%$ del entrenador 2 y el $14,01 \%$ del entrenador 3 . Se puede deducir que esta poca receptividad a la formación puede ser debida a estilos interpersonales muy arraigados, en los que los cambios en estadíos comportamentales requieran de un mayor tiempo de formación y supervisión o seguimiento de las conductas verbales que se desean modificar.

Otro estudio con resultados similares (Conde, Almagro, Sáenz-López, Domínguez, y MorenoMurcia, 2010) desarrollado con cinco entrenadores de baloncesto con una titulación mínima de nivel I, tras tres meses de supervisión reflexiva cada 15 días, obtuvo una mejora significativa en los entrenadores del grupo experimental. Esto demuestra que los entrenadores con formación específica tienen mayor predisposición a introducir cambios en su metodología de intervención. El hecho de que en este estudio (Conde et al., 2010) los entrenadores hayan conseguido una mejora significativa, puede ser debido a la interacción de dos factores importantes: la cualificación de los entrenadores y el tiempo de impartición del programa, reforzado por la supervisión reflexiva por parte de expertos.

\section{CONCLUSIONES}

Tras la investigación, en base a los resultados obtenidos y a la bibliografía consultada, podemos concluir que el programa de formación ha tenido un efecto positivo en dos de los cuatro entrenadores evaluados, un efecto neutro en el entrenador 3 y un efecto no deseado en el entrenador 4. Por tanto, el programa resulta eficaz parcialmente, por lo que resulta necesario seguir investigando y profundizando en esta línea de trabajo.

\section{APLICACIONES PRÁCTICAS}

En un futuro, sería conveniente aumentar las horas de formación del programa. Además, sería interesante aplicar el programa a entrenadores con formación, bien académica o federativa, o incluso a entrenadores con ambas formaciones y realizar comparaciones de cómo influye entre entrenadores con formación y entrenadores sin formación. Por otro lado, consideramos adecuado extender el campo de aplicación a otros deportes como el baloncesto o el balonmano, e incluso llevar a cabo un estudio con entrenadoras.

También, cabe destacar como posible limitación la reducida muestra participante, hecho que se justifica en base a la dificultad del proceso en sí, tanto a lo referente a la etapa de diseño como a la de recogida y análisis de datos al tratarse de una observación real del comportamiento del entrenador, no basada en cuestionarios de percepción. Otra posible limitación, es la falta de seguimiento o intervenciones tras el programa de formación desarrollado, es decir, no realizar exclusivamente grabaciones y recogida de datos y llevar a cabo valoraciones y propuestas de mejora de manera individualizada para cada técnico en los entrenamientos y partidos.

\section{AGRADECIMIENTOS}

Fondo Social Europeo y la Consejería de Economía e Infraestructuras - Secretaría General de Ciencias, Tecnología e Innovación (Junta de Extremadura): "PO17012".

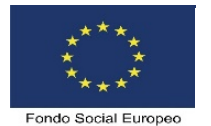

JUNTA DE EXTREMADURA

Consejería de Economía e Infraestructuras

\section{REFERENCIAS}

1. Adie, J.W., Duda, J.L. y Ntoumanis, N. (2012). Perceived coach-autonomy support, basic need satisfaction and the well-and ill-being of elite youth soccer players. A longitudinal investigation. Psychology of Sport and Exercise, 13, 51-59. 
https://doi.org/10.1016/j.psychsport.2011.07.008

2. Anguera, M.T., Blanco-Villaseñor, A., Hernández-Mendo, A. y Losada, J.L. (2011). Diseños observacionales: ajuste y aplicación en psicología del deporte. Cuadernos de Psicología del Deporte, 11(2), 63-76.

3. Anguera, M.T. y Hernández-Mendo, A. (2014). Metodología observacional y psicología del deporte: Estado de la cuestión. Revista de Psicología del Deporte, 23(1), 103-109. https://ddd.uab.cat/record/119077

4. Balaguer, I., González L., Fabra, P., Castillo, I., Mercé, J. y Duda, J.L. (2012). Coaches' interpersonal style, basic psychological needs and the well- and ill-being of young soccer players: A longitudinal analysis. Journal of Sport Sciences, 30(15), 1619-1629. https://doi.org/10.1080/02640414.2012.731517

5. Bartholomew, K.J., Ntoumanis, N. y ThøgersenNtoumani, C. (2009). A review of controlling motivational strategies from a self-determination theory perspective: implications for sport coaches. International Review of Sport and Exercise Psychology, 2, 215-233. https://doi.org/10.1080/17509840903235330

6. Castillo, I., González L., Fabra, P., Mercé, J. y Balaguer, I. (2012). Estilo interpersonal controlador del entrenador, frustración de las necesidades psicológicas básicas, y burnout en futbolistas infantiles. Cuadernos de Psicología del Deporte, 12(1), 143-146. https://doi.org/10.4321/S1578-

84232012000100014

7. Cecchini, J.A., Méndez-Jiménez, A. y Fernández-Rio, J. (2014). Meta-percepciones de competencia de terceros significativos, competencia percibida, motivación situacional y orientaciones de deportividad en jóvenes deportistas. Revista Psicología del Deporte, 23(2), 285-293. https://ddd.uab.cat/record/119428

8. Conde, C., Almagro, B.J., Sáenz-López, P., Domínguez, A. y Moreno-Murcia, J.A. (2010). Evaluación e influencia de un programa de formación orientado a la mejora del clima motivacional transmitido por entrenadores de baloncesto. European Journal of Human Movement, 25, 165-182.

9. Cruz, J., Torregrosa, M., Sousa, C., Mora, A. y Viladrich, C. (2010). Efectos conductuales de programas personalizados de asesoramiento a entrenadores en estilo de comunicación y clima motivacional. Revista de Psicología del Deporte, 20(1), 179-195.

10. Deci, E.L. y Ryan, R.M. (1985). Intrinsic motivation and self-determination in human behavior. New York: Plenum Press. https://doi.org/10.1007/978-1-4899-2271-7

11. Deci, E.L. y Ryan, R.M. (2000). Target Article: The "What" and "Why" of goal pursuits: Human needs and the self-determination of behavior. Psychological Inquiry, 11, 227-268. https://doi.org/10.1207/S15327965PLI1 104_01

12. Duda, J.L., Quested, E., Haug, E., Samdal, O., Wold, B., Balaguer, I. ... y Cruz, J. (2013). Promoting Adolescent health through and intervention aimed at improving the quality of their participation in Physical Activity (PAPA): Background to the project and main trial protocol. International Journal of Sport and Exercise Psychology, 11, 319-327. https://doi.org/10.1080/1612197X.2013.839413

13. Feu, S., Ibáñez, S.J. y Gozalo, M., (2010). Influencia de la formación formal y no formal en las orientaciones que adoptan los entrenadores deportivos. Revista de Educación, 353, 615-640.

14. Fraile, A., de Diego Vallejo, R. y Boada i Grau, J. (2011). El perfil de los técnicos del deporte escolar en un contexto europeo. Revista Internacional de Medicina y Ciencias de la Actividad Física y el Deporte, 11(42), 278-297. http://cdeporte.rediris.es/revista/revista42/artperf il205.htm

15. Galván, J.F., López-Walle, J.M., Pérez-García, J. A., Tristán, J.L. y Medina-Rodríguez, R.E. (2013). Clima motivacional en deportes individuales y de conjunto en atletas jóvenes mexicanos. Revista Iberoamericana de Psicología del Ejercicio y el Deporte, 8(2), 393410.

16. García-Calvo, T., Sánchez-Oliva, D., Leo, F., Amado, D. y Pulido, J.J. (2016). Effects of an intervention programme with teachers on the development of positive behaviours in Spanish physical education classes. Physical Education and Sport Pedagogy, 21, 527-588. https://doi.org/10.1080/17408989.2015.1043256

17. Haerens, L., Aelterman, N., Van den Berghe, L., De Meyer, J., Soenens, B. y Vansteenkiste, M. (2013). Observing Physical Education Teachers' 
Pulido, J. J. (1), Merino, A. (2), Sánchez-Oliva, D. (3), Tapia, M. A. (4), y García-Calvo, T. (5)

Need-Supportive Interactions in Classroom Settings. Journal of Sport \& Exercise Psychology, 35, 3-17. https://doi.org/10.1123/jsep.35.1.3

18. Jowett, S. y Cockerill, I.M. (2003). Olympic medalists' perspective of the athlete-coach relationship. Psychology of Sport and Exercise, 4, 313-331.

https://doi.org/10.1016/S1469-0292(02)00011-0

19. Kenneth, G., More, K.G. y Franks, I.M. (1996). Analysis and modification of verbal coaching behaviour: The usefulness of a data-driven intervention strategy. Journal of Sports Sciences, 14 , 523-543. https://doi.org/10.1080/026404196367651

20. Landis, J.R., y Koch, G.G. (1977). The measurement of observer agreement for categorical data. Biometrics, 33(1), 159-174. https://doi.org/10.2307/2529310

21. Leo, F., García-Calvo, T., Sánchez-Miguel, P.A., Sánchez-Oliva, D. y Gómez, F. (2010). Análisis en las diferencias de la conducta verbal entre entrenadores de fútbol con formación y sin formación universitaria en la iniciación deportiva. Kronos, 17, 81-88.

22. Mesquita, I., Farias, C., Rosado, A., Pereira, F. y Moreno, M.P. (2008). La presentación de las tareas en función de la formación académica de los entrenadores de fútbol. European Journal of Human Movement, 20, 128-143.

23. Morelló E., Vert, B. y Navarro, S. (2018).

Establecimiento de objetivos en el currículum formativo de los futbolistas. Revista de Psicología Aplicada al Deporte y al Ejercicio Físico, 3, 1-9.

http://dx.doi.org/10.5093/rpadef2018a7

24. Portney, L.G. y Watkins, M.P. (2009). Statistical measures of validity. Foundations of clinical research: Applications to practice. $3^{\text {a }}$ ed. Upper Saddle River (Nueva Jersey): Pearson/Prentice Hall, 644-53.

25. Prieto, J.M. (2016). Relación entre competitividad, ansiedad social y compromiso con variables deportivas y académicas en futbolistas jóvenes. Revista Iberoamericana de Psicología del Ejercicio y el Deporte, 11(2), 193200.

26. Pulido J.J., Leo, F., Chamorro, J.M. y GarcíaCalvo, T. (2015). ¿Apoyan los entrenadores la motivación de sus deportistas? Diferencias en la percepción del comportamiento. Revista de Psicología del Deporte, 24(1), 139-145.

27. Pulido, J.J., Sánchez-Oliva, D., Sánchez-Miguel, P.A., Leo, F.M. y García-Calvo, T. (2016). Influencia de la formación de los entrenadores sobre la motivación de los deportistas. Revista Internacional de Medicina y Ciencias de la Actividad Física y el Deporte, 16(64), 685-702. http://dx.doi.org/10.15366/rimcafd2016.64

28. Pulido, J.J., Sánchez-Oliva, D., Leo, F.M., Matos, S. y García-Calvo, T. (2017). Effects of an Interpersonal Style Intervention for Coaches on Young Soccer Players' Motivational Processes. Journal of Human Kinetics, 59(1), 107-120.

https://dx.doi.org/10.1515\%2Fhukin-2017-0151

29. Pulido, J.J., Sánchez-Oliva, D., Silva, M., Palmeira, A. y García-Calvo, T. (en revisión). Development and validation of the Coach Interpersonal Style Observational System (CISOS). Journal of Sport and Exercise Psychology.

30. Ramis, Y., Torregrosa, M., Viladrich, C. y Cruz, J. (2013). El apoyo a la autonomía generado por entrenadores, compañeros y padres y su efecto sobre la motivación autodeterminada de deportistas de iniciación. Anales de Psicología, 29(1), 243-248. http://dx.doi.org/10.6018/analesps.29.1.124011

31. Sánchez-Oliva, D., Leo, F.M., Sánchez-Miguel, P.A., Amado, D. y García-Calvo, T. (2010). Análisis de la conducta verbal del entrenador de fútbol en función de su formación federativa y del período del partido en categorías inferiores. Retos. Nuevas Tendencias en Educación Física, Deporte y Recreación, 18, 24-28.

32. Smith, R.E., Smoll, F.L. y Cumming, S.P. (2007). Effects of a motivational climate intervention for coaches on young athletes'sport performance anxiety. Journal of Sport and Exercise Psychology, 29(1), 39-59. https://doi.org/10.1123/jsep.29.1.39

33. Smith, R.E., Smoll, F.L. y Curtis, B. (1979). Coach efectiveness training. A cognitive behavioral approach to enhancing relationship skills in youth sports coaches. Journal of Sport Psychology, 1, 59-75. https://doi.org/10.1123/jsp.1.1.59

34. Soriano, G., Ramis, Y, Cruz, J. y Sousa, C. (2014). Un programa de intervención 
individualizado con entrenadores de fútbol. Cuadernos de Psicología del Deporte, 14(3), 99$106 . \quad$ https://doi.org/10.4321/S157884232014000300011

35. Sousa, C., Cruz, J., Torregrosa, M., Vilches, D. y Viladrich, C. (2006). Evaluación conductual y Programa de Asesoramiento Personalizado a Entrenadores (PAPE) de deportistas jóvenes. Revista de Psicología del Deporte, 15(2), 263278.

36. Sousa, C., Cruz, J., Viladrich, C. y Torregrosa, M. (2007). Efectos del programa de asesoramiento personalizado a entrenadores (PAPE) en el compromiso deportivo y el abandono de futbolistas jóvenes. European Journal of Human Movement, 19, 97-116.

37. Tessier, D., Smith N., Tzioumakis, Y., Quested, E., Sarrazin, P., Papaioannou, A., Digelidis, N. y Duda, J.L. (2013). Comparing the objective motivational climate created by grassroots soccer coaches in England, Greece and France. International Journal of Sport and Exercise Psychology, 11(4), 365-383.

https://dx.doi.org/10.1080/1612197X.2013.8312 59

38. Van den Berghe, L., Soenens, B., Vansteenkiste, M., Aelterman, N., Cardon, G., Tallir, I.B. y Haerens, L. (2013). Observed need-supportive and need-thwarting teaching behavior in physical education: Do teachers' motivational orientations matter? Psychology of Sport and Exercise, 14(5), 650-661.

https://doi.org/10.1016/j.psychsport.2013.04.006 